\title{
Lobar Bronchus
}

National Cancer Institute

\section{Source}

National Cancer Institute. Lobar Bronchus. NCI Thesaurus. Code C32998.

A part the bronchial tree, arising from the primary bronchi, with each one serving as the airway to a specific lobe of the lung. 